\title{
Combinatorics of diagrams of permutations
}

\author{
Joel Brewster Lewis I $^{*}$ and Alejandro H. Morales $\|^{2}$ \\ ${ }^{1}$ University of Minnesota, Minneapolis, MN 55455, USA \\ ${ }^{2}$ LaCIM, Université du Québec à Montréal, Canada
}

\begin{abstract}
There are numerous combinatorial objects associated to a Grassmannian permutation $w_{\lambda}$ that index cells of the totally nonnegative Grassmannian. We study some of these objects (rook placements, acyclic orientations, various restricted fillings) and their $q$-analogues in the case of permutations $w$ that are not necessarily Grassmannian.

Résumé. Il y a nombreaux objets combinatoires associés à une permutation Grassmannienne $w_{\lambda}$ qui indexent les cellules de le Grassmannien totalement non négatif. Nous étudions certains de ces objets (placement de tours, orientations acycliques, diverses remplissages restrentes) et leurs $q$-analogues dans le cas où la permutation $w$ n'est pas nécessairement Grassmannienne.
\end{abstract}

Keywords: Permutation diagram, acyclic orientation, Grassmannian, $q$-analogue, rook placement, Le diagram

\section{Introduction}

In his study [9] of the totally nonnegative Grassmannian $G r_{k, n}^{\geq 0}(\mathbb{R})$, Postnikov introduced a "zoo" of combinatorial objects that parametrize cells of the matroidal decomposition of $G r_{k, n}^{\geq 0}(\mathbb{R})$. This decomposition refines the Schubert decomposition $G r_{k, n}=\cup_{\lambda \subset\left\langle(n-k)^{k}\right\rangle} \Omega_{\lambda}$, and the members of the zoo are most easily identified with the Grassmannian permutations $w=w_{\lambda} \in \mathfrak{S}_{n}$. Of these combinatorial objects, we are particularly interested in the following five (all of which will be defined later); see Figure 1 for examples.

(i) The set $\mathcal{A O}_{w}$ of acyclic orientations of the inversion graph $G_{w}$. We write $A O_{w}=\# \mathcal{A O}{ }_{w}$.

(ii) The set $\mathcal{R} \mathcal{P}_{w}$ of placements of $n$ non-attacking rooks on the complement $\overline{O_{w}}:=([n] \times[n]) \backslash O_{w}$ of the $S W$ diagram $O_{w}$ of $w$. We write $R P_{w}=\# \mathcal{R} \mathcal{P}_{w}$.

(iii) The set of Gamma fillings of the $S E$ diagram $E_{w}$. We denote the number of such fillings by $\Gamma_{w}$.

(iv) The set of $L$ fillings of SE diagram $E_{w}$. We denote the number of such fillings by $\mathrm{L}_{w}$.

(v) The set $[i d, w]=\left\{u \in \mathfrak{S}_{n} \mid i d \preceq u \preceq w\right\}$ of permutations below $w$ in the strong Bruhat order. The work of Postnikov [9, Thm. 24.1] and Spiridonov [11, §3] establishes the following result.

Theorem 1.1. For a Grassmannian permutation $w_{\lambda}$ in $\mathfrak{S}_{n}$, the numbers $A O_{w_{\lambda}}, R P_{w_{\lambda}}, \Gamma_{w_{\lambda}}, \mathrm{L}_{w_{\lambda}}$, and $\#\left[i d, w_{\lambda}\right]$ are equal.

This theorem naturally raises the following question:

${ }^{*}$ Supported by NSF RTG grant NSF/DMS-1148634

${ }^{\dagger}$ Supported by a CRM-ISM Postdoctoral Fellowship. A portion of this work was done during a two month stay at ICERM. 
Problem 1.2. Characterize the relation among these five numbers when $w$ is not Grassmannian.

In the rest of this introduction we give background on previous work and a summary of our own results towards answering this problem and its refinements.

\subsection{Definitions}

We begin by giving the definitions of the terms in the preceding paragraphs, which will be used throughout this extended abstract. Let $w=w_{1} w_{2} \ldots w_{n}$ be a permutation in the symmetric group $\mathfrak{S}_{n}$ on $n$ letters. The pair $(i, j)$ is said to be an inversion of $w$ if $1 \leq i<j \leq n$ and $w_{i}>w_{j}$. The inversion graph $G_{w}$ of $w$ is the graph with vertex set $[n]:=\{1,2, \ldots, n\}$ and with edges given by the inversions of $w$. An acyclic orientation of a graph $G$ is an orientation of the edges of $G$ so that the oriented graph has no directed cycles.

The (strong) Bruhat order $\preceq$ on $\mathfrak{S}_{n}$ is the partial order on the symmetric group defined by the cover relations $w \prec w \cdot t_{i j}$ if $\ell\left(w \cdot t_{i j}\right)=\ell(w)+1$ and $t_{i j}$ is the transposition that switches $i$ and $j$.

We say that a permutation $w_{\lambda}$ in $\mathfrak{S}_{n}$ is a Grassmannian permutation if it has a unique descent; say the position of the descent is $k$. Each such permutation is associated to a partition $\lambda$ inside the $k \times(n-k)$ box $\left\langle(n-k)^{k}\right\rangle$ (i.e., a partition with at most $k$ parts and largest part at most $n-k$ ).

A diagram is a finite subset of $\mathbb{Z}_{>0} \times \mathbb{Z}_{>0}$. The south-east (SE) diagram $E_{w}$ (respectively, southwest (SW) diagram $O_{w}$ ) of the permutation $w$ is the subset of $[n] \times[n]$ consisting of those elements not directly to the south or east (respectively, south or west) of an entry in the permutation matrix of $w$. The size of $E_{w}$ is the number $\ell(w)$ of inversions of $w$, while the size of $O_{w}$ is the number of anti-inversions, i.e., the number of pairs $(i, j)$ such that $1 \leq i<j \leq n$ and $w_{i}<w_{j}$. (This is also $\left(\begin{array}{l}n \\ 2\end{array}\right)-\ell(w)$.)

A Gamma filling ${ }^{(i)}$ of a diagram $D$ is an assignment of 0 s and 1 s to the elements of $D$ subject to the condition that there are no four entries in $D$ at the vertices of a (axis-aligned) rectangle with either of the following fillings: \begin{tabular}{ll}
\hline $\begin{array}{l}0 \\
1\end{array}$ & 1
\end{tabular}, $\begin{array}{lll}0 & 1 \\
1 & 0\end{array}$. Similarly, an $\mathbf{L}$ filling of $D$ is an assignment of 0 s and 1 s to the elements

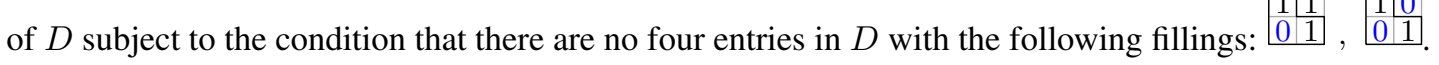
Example 1.3. For the Grassmannian permutation $w=3412$, the SW diagram $O_{3412}$ consists of the two elements $(1,4),(3,2)$ and the SE diagram $E_{3412}$ consists of the four elements $(1,1),(1,2),(2,1),(2,2)$. The inversion graph $G_{3412}$ has four edges. See Figure 1(a) For $w=3142$, the SW diagram $O_{3142}$ and the SE diagram $E_{3142}$ have three elements each and the inversion graph $G_{3142}$ has three edges. This $w$ is not Grassmannian, and its SE diagram is not the diagram of a partition in French notation. See Figure 1(b).

\subsection{Previous work}

The first result in the direction of Problem 1.2 was the paper [3] of Hultman-Linusson-ShareshianSjöstrand settling a conjecture of Postnikov [9, Conj. 24.4]. Their result explains the relation between the number $A O_{w}$ of acyclic orientations of the inversion graph of $w$ and the size of the Bruhat interval $[i d, w]$ (numbers (i) and (v) above).

Theorem 1.4 (Hultman-Linusson-Shareshian-Sjöstrand [3]). For all permutations $w$ in $\mathfrak{S}_{n}$, we have $A O_{w} \leq \#[i d, w]$. Furthermore, equality holds if and only if $w$ avoids the permutation patterns 4231, 35142,42513 , and 351624.

\footnotetext{
(i) In [9], Postnikov used English notation for partitions, while we use French notation; thus, our Gamma diagrams are the equivalent of his "Le" diagrams.
} 


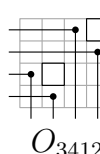

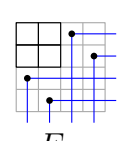

$E_{3412}$

(a)

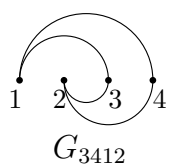

$G_{3412}$

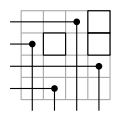

$\mathrm{O}_{3142}$

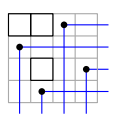

$E_{3142}$

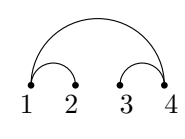

$G_{3142}$

(b)

Fig. 1: The SW diagram, the SE diagram, and the inversion graph of (a) $w=3412$ and (b) $u=3142$. For the Grassmannian permutation $w=3412$ (associated to the shape $\lambda=\langle 2,2\rangle$ ) we have $A O\left(G_{3412}\right)=R P_{3412}=$ $\#[1234,3412]=\Gamma_{3412}=\mathrm{L}_{3412}=14$.

(The permutations on which equality is achieved are very special, and will appear in the sequel. We call them Gasharov-Reiner permutations after their first appearance [2] in the literature.)

Another result, relating (iv) and (v) above, was proved by Postnikov and Spiridonov (private communication). When $w=w_{\lambda}$ is Grassmannian the diagram $E_{w}$ is (up to removing empty rows and columns) the Young diagram of $\lambda$ in French notation. Such permutations are also those that avoid the permutation patterns 321 and 2143. It is well known that when $w$ just avoids 321 the diagram $E_{w}$ is a skew Young diagram $\lambda / \mu$ (in French notation).

Theorem 1.5 (Postnikov-Spiridonov (unpublished)). If $w$ avoids 321 then the number $\mathrm{L}_{w}$ of $\mathrm{L}$-fillings of $E_{w}$ equals \# $[i d, w]$.

Various authors have also explored $q$-analogues of the various objects defined above. In particular, Oh-Postnikov-Yoo established [8, Thm. 7] the following result linking a $q$-analogue $A_{w}(q)$ (whose definition we omit) of $A O_{w}$ to the Poincaré polynomial $P_{w_{\lambda}}(q)=\sum_{u \preceq w_{\lambda}} q^{\ell(u)}$ (here the sum is over the permutations $u$ in the Bruhat interval $\left[i d, w_{\lambda}\right]$ ).

Theorem 1.6 (Oh-Postnikov-Yoo [8, Thm. 7]). For a permutation $w$ in $\mathfrak{S}_{n}, A_{w}(q)=P_{w}(q)$ if and only if $w$ avoids 3412 and 4231 .

(Note that the class of permutations of Theorem 1.4 and Theorem 1.6 differ, thus the $q=1$ version of the latter does not imply the former.)

\subsection{New results}

In Section 2, we continue the study of the relationships between the objects in Theorem 1.1 for general permutations. Our first result (which was independently obtained by Hultman) is an equivalence among the number of acyclic orientations of the inversion graph $G_{w}$ and rook placements on $\overline{O_{w}}$.

Theorem 2.1. Given any permutation $w$ in $\mathfrak{S}_{n}$, the following are equal: the number $A O_{w}$ of acyclic orientations of the inversion graph $G_{w}$, and the number $R P_{w}$ of placements of n non-attacking rooks on the complement of the $S W$ diagram $O_{w}$.

An immediate consequence of Theorem 1.4 and Theorem 2.1 is that the number $R P_{w}$ of rook placements has the same relation with $\#[i d, w]$ as the number $A O_{w}$ of acyclic orientations (see Corollary 2.2. Another consequence (when combined with work of Spiridonov [11] and Josuat-Vergès [4, §4]) is that when $w$ avoids 321 then the number $\Gamma_{w}$ of $\Gamma$-fillings on the SE diagram $E_{w}$ of $w$ equals the number $A O_{w}$ of acyclic orientations (see Corollary 2.12). 
In Section 3. we study relations among $q$-analogues of the objects studied above. One of these, the Poincaré polynomial $P_{w}(q)$, is defined in the previous section. The other is a natural $q$-analogue of the rook placements $\mathcal{R} \mathcal{P}_{w}$ on the SW diagram of $w$ :

(ii) $q$ Let $\mathbf{F}_{q}$ be the finite field with $q$ elements. Define mat $_{w}(q)$ be the number of $n \times n$ invertible matrices in $\mathbf{F}_{q}$ whose nonzero entries are in $\overline{O_{w}}$.

It was shown in [6, Prop. 5.1] that $\operatorname{mat}_{w}(q) /(q-1)^{n}$ is an enumerative $q$-analogue of $R P_{w}$, that is

$$
\operatorname{mat}_{w}(q) /(q-1)^{n} \equiv R P_{w}\left(=A O_{w}\right) \quad(\bmod q-1) .
$$

Remarkably, the equality condition between $\operatorname{mat}_{w}(q) /(q-1)^{n}$ and (an appropriately rescaled version of) $P_{w}(q)$ is precisely the same as between their values at $q=1$ (as in Theorem 1.4). This settles part of a conjecture [5, Conj. 6.6] of Klein and the present authors.

Theorem 3.1. Let $w$ be a permutation in $\mathfrak{S}_{n}$ and let $O_{w}$ be the $S W$ diagram of $w$. Then $\operatorname{mat}_{w}(q) /(q-$ $1)^{n}=q^{\left(\begin{array}{c}n \\ 2\end{array}\right)+\ell(w)} P_{w}\left(q^{-1}\right)$ if and only if $w$ avoids the patterns $4231,35142,42513$, and 351624 .

\section{Acyclic orientations and rook placements on diagrams of per- mutations}

The main result of this section is the following:

Theorem 2.1. Given a permutation $w$ in $\mathfrak{S}_{n}$, the following are equal: the number $A O_{w}$ of acyclic orientations of the inversion graph of $w$, the number $R P_{w}$ of placements of n non-attacking rooks on the complement of the $S W$ diagram $O_{w}$ of $w$, and the number of "percentage pseudo-avoiding fillings" of the $S E$ diagram $E_{w}$ of $w$.

As corollaries of Theorem 2.1 we obtain the following results. First, using Theorem 1.4, we have:

Corollary 2.2. The number of placements of n non-attacking rooks on $\overline{O_{w}}$ equals the number of permutations in the Bruhat interval [id, $w]$ if and only if $w$ avoids 4231, 35142, 42513, and 351624.

Second, recall that when $w$ avoids 321 , the diagram $E_{w}$ is a skew Young diagram. By a result of Spiridonov [11] and Josuat-Vergès [4, \$4] the number of such fillings' of skew Young diagrams equals the number of $\Gamma$-fillings. Thus we also obtain the following result:

Corollary 2.12. If $w$ avoids 321 then the number of L-diagrams on $E_{w}$ is equal to the number of acyclic orientations of the inversion graph of $w$.

In Section 2.1 we prove the equality of $A O_{w}$ and $R P_{w}$, and in Section 2.2 we define the percentage pseudo-avoiding fillings and complete the proof of Theorem 2.1

Remark 2.3. It is relatively easy to prove a special case of Theorem 2.1 when the reverse of $w$ is vexillary, i.e., when $w$ avoids 3412 . In this case $\overline{O_{w}}$ is, up to permuting rows and columns, a Young diagram. Then calculating the number of rook placements is easy: given a Young diagram $\lambda=\left(\lambda_{1}, \lambda_{2}, \ldots, \lambda_{n}\right)$ where $\lambda_{1} \leq \lambda_{2} \leq \cdots$, the number of placements of $n$ non-attacking rooks on $\lambda$ is just $\prod_{i=1}^{n}\left(\lambda_{i}-i+1\right)$. On the other hand, we say that a graph is chordal if every cycle of four or more edges in the graph has a chord, i.e., an edge joining two non-consecutive vertices in the cycle. The chromatic polynomial of a chordal graph $G$ with $n$ vertices is of the form $\chi_{G}(t)=\prod_{i=1}^{n}\left(t-e_{i}\right)$. Therefore the number of acyclic orientations of $G$ is $(-1)^{n} \chi_{G}(-1)=\prod_{i=1}^{n}\left(e_{i}+1\right)$. One can show that the inversion graph $G_{w}$ is chordal if and only if $w$ avoids 3412 and that in this case the multisets $\left\{e_{i}\right\}_{i=1}^{n}$ and $\left\{\lambda_{i}-i\right\}_{i=1}^{n}$ are equal. 


\subsection{Deletion-contraction for diagrams and inversion graphs}

In this section, we prove equality of the first two objects in Theorem 2.1. It is well-known that to count acyclic orientations of a graph, one may use deletion-contraction relations. Our proof has two steps. First, we show that when the graph in question is the inversion graph of a permutation, we may choose the edge to be deleted or contracted in such a way that the resulting graphs are also inversion graphs of permutations. Second, we show that rook placements avoiding SW diagrams obey the same recursion by considering whether or not a rook is placed on a particular (carefully chosen) cell of the board. (The steps of this argument are illustrated in Figure 2, ) Then the desired equality will follow by induction. We break the proof up into several smaller results, beginning with the base case of the induction.

Proposition 2.4. For the identity permutation $i d$, the number of rook placements $R P_{i d}$ avoiding the $S W$ diagram $O_{i d}$ is equal to the number $A O_{i d}$ of acyclic orientations of the inversion graph $G_{i d}$.

Proof: The diagram $O_{i d}$ consists of all entries above the main diagonal and so $i d$ is the unique rook placement avoiding $O_{i d}$, while the graph $G_{i d}$ is empty and so has a unique (trivial) acyclic orientation.

In what follows, we will use the following conventions for the action of permutations on permutations, graphs, and diagrams: multiplying a permutation $w \in \mathfrak{S}_{n}$ on the left by the adjacent transposition $s_{i}$ switches the letters in the $i$ th and $(i+1)$ th positions; the action of $s_{i}$ on a graph with vertex set $[n]$ is to switch the vertices $i$ and $i+1$; and multiplying a diagram on the left by $s_{i}$ interchanges the $i$ th and $(i+1)$ th rows.

Now choose a permutation $w=w_{1} \cdots w_{n} \in \mathfrak{S}_{n}$ other than the identity permutation, and let $i$ be minimal such that $w_{i}>w_{i+1}$. Then $e=(i, i+1)$ is an edge in $G_{w}$, while $\left(i+1, w_{i}\right)$ is an entry of $[n] \times[n]$ not in $O_{w}$. It is this edge and this position that will be the focus of our recursion, deleting and contracting or deciding whether or not to place a rook. We begin with the easy cases; first, deletion of the edge $e$.

Proposition 2.5. Given $w \in \mathfrak{S}_{n}$ with first descent at position $i$, the graphs $G_{s_{i} w}$ and $G_{w} \backslash\{(i, i+1)\}$ are isomorphic and in particular have the same number of acyclic orientations.

Proof: It's easy to see that $G_{s_{i} w}=s_{i}\left(G_{w} \backslash\{(i, i+1)\}\right)$, i.e., the graph isomorphism is "interchange the $i$ th and $(i+1)$ th vertices."

The corresponding reduction for diagrams is to choose not to place a rook in position $\left(i+1, w_{i}\right)$.

Proposition 2.6. Given $w \in \mathfrak{S}_{n}$ with first descent at position $i$, rook placements on $[n] \times[n]$ avoiding $O_{w}$ in which no rook is placed in position $\left(i+1, w_{i}\right)$ are in bijection with rook placements avoiding $O_{s_{i}} w$.

Proof: Rook placements with no rook in position $\left(i+1, w_{i}\right)$ are precisely those placements avoiding $O_{w} \cup\left\{\left(i+1, w_{i}\right)\right\}$; moreover, this diagram is exactly equal to $s_{i} \cdot O_{s_{i} w}$. Since the number of rook placements is unchanged by switching rows and columns, this gives the desired result.

Now we consider the harder cases: in the case of graphs, this is contraction, while in the case of diagrams it is the forced choice of a rook.

Given $w \in \mathfrak{S}_{n}$ with first descent in position $i$. Let $j$ be minimal so that $w_{j}>w_{i+1}$. Let $w^{\prime} \in \mathfrak{S}_{n-1}$ be the permutation order-isomorphic to

$$
w_{1} w_{2} \cdots w_{j-1} w_{i} w_{j} w_{j+1} \cdots w_{i-1} w_{i+2} w_{i+3} \cdots w_{n}
$$



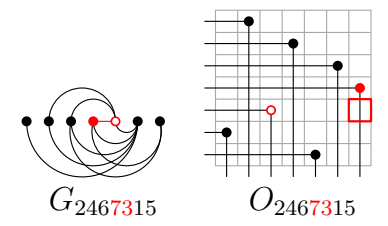

(a)

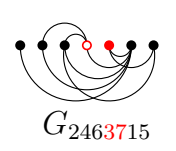

(b)

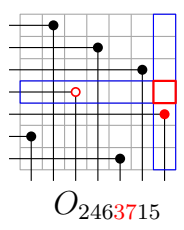

b)

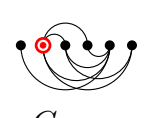

$G_{263514}$

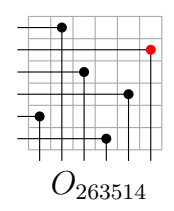

(c)

Fig. 2: (a) inversion graph and diagram, (b) inversion graph and diagram for deletion, (c) inversion graph and diagram for contraction.

Proposition 2.7. With $w, i, w^{\prime}$ as above, we have that the contracted graph $G_{w} / e$ is isomorphic to the inversion graph $G_{w^{\prime}}$, and so in particular the two graphs have the same number of acyclic orientations.

Proof sketch: The isomorphism identifies the contracted vertex in $G_{w} / e$ with the vertex in $G_{w^{\prime}}$ that is the image (under order-isomorphism) of the entry $w_{i}$, and otherwise identifying vertices in the obvious way. One must carefully check in several cases that this preserves edges.

Now we give the analogous result for diagrams. For $w \in \mathfrak{S}_{n}$ with first descent at position $i$, define the contracted diagram $O_{w} /(i, i+1) \subset[n-1] \times[n-1]$ to be the result of removing the $(i+1)$ th row and $w_{i}$ th column from $O_{w}$, sliding rows to the left and up as necessary. We have the following trivial result.

Proposition 2.8. The number of rook placements on $[n] \times[n]$ avoiding $O_{w}$ in which a rook is placed in position $\left(i+1, w_{i}\right)$ is equal to the number of rook placements on $[n-1] \times[n-1]$ avoiding the diagram $O_{w} /(i, i+1)$.

The next step is to show that the permutation $w^{\prime}$ defined earlier has a SW diagram essentially equal to $O_{w} /(i, i+1)$. The proof is omitted.

Proposition 2.9. With $w, i, w^{\prime}$ as above, we have that the diagram $O_{w^{\prime}}$ is the same as the diagram $O_{w} / e$ up to row and column switches, and so in particular $R P\left(O_{w^{\prime}}\right)=R P\left(O_{w} / e\right)$.

Finally, putting all these results together we get the main result of this section:

Theorem 2.1. Given a permutation $w \in \mathfrak{S}_{n}$, the number of rook placements on $[n] \times[n]$ avoiding $O_{w}$ is equal to the number of acyclic orientations of the inversion graph $G_{w}$.

Proof: It follows from Propositions 2.5 and 2.7 and usual deletion-contraction that

$$
A O_{w}=A O_{s_{i} w}+A O_{w^{\prime}}
$$

and it follows from Propositions 2.6 and 2.9 and the fact that every rook placement avoiding $O_{w}$ either does or does not have a rook at position $\left(i+1, w_{i}\right)$ that

$$
R P_{w}=R P_{s_{i} w}+R P_{w^{\prime}}
$$

Since $s_{i} w$ has strictly fewer inversions than $w$, the result follows by induction (with Proposition 2.4 as a base case). 
Remark 2.10. Note that if $\chi_{G}(t)$ is the chromatic polynomial of the graph $G$ with $n$ vertices, then $A O(G)=(-1)^{n} \chi_{G}(-1)$. By deletion-contraction, an analogue of (2) holds for the chromatic polynomial of $G_{w}$, namely

$$
\chi_{G_{w}}(t)=\chi_{G_{s_{i} w}}(t)-\chi_{G_{w^{\prime}}}(t) .
$$

Is there a $q$-analogue of (3)? And if so is there a common $q, t$ refinement of it with (4)? Note that in Section 3 we will give $q$-analogues of (3) that hold only for certain permutations (see Theorem 3.1).

\subsection{Acyclic orientations and percentage pseudo-avoiding fillings}

Recall that $E_{w}$ is the subset of $[n] \times[n]$ consisting of all pairs $\left(i, w_{j}\right)$ such that $i<j$ and $w_{j}<w_{i}$ (see Figure 1(a) center). In this section, we complete the proof of Theorem 2.1 by establishing the equality of the number $A O_{w}$ of acyclic orientations of the inversion graph of $w$ with the number of percentage pseudo-avoiding fillings of the SE diagram $E_{w}$, which we define now.

Given a permutation $w$, we say that a filling $A$ of $E_{w}$ with 0 s and $1 \mathrm{~s}$ is a percentage pseudo-avoiding filling if it satisfies the following conditions:

(i) if squares $(i, j),\left(i^{\prime}, j\right),\left(i, j^{\prime}\right)$ and $\left(i^{\prime}, j^{\prime}\right)$ are elements of $E_{w}$ then we do not have $A_{i, j}=A_{i^{\prime}, j^{\prime}}=1$ and $A_{i^{\prime}, j}=A_{i, j^{\prime}}=0$, nor do we have $A_{i, j}=A_{i^{\prime}, j^{\prime}}=0$ and $A_{i^{\prime}, j}=A_{i, j^{\prime}}=1$;

(ii) if squares $(i, j),\left(i^{\prime}, j\right)$ and $\left(i, j^{\prime}\right)$ are elements of $E_{w}$ and square $\left(i^{\prime}, j^{\prime}\right)$ is an entry of $w$ (i.e., of the form $\left.\left(k, w_{k}\right)\right)$ then we do not have $A_{i, j}=1$ and $A_{i^{\prime}, j}=A_{i, j^{\prime}}=0$, nor do we have $A_{i, j}=0$ and $A_{i^{\prime}, j}=A_{i, j^{\prime}}=1$.

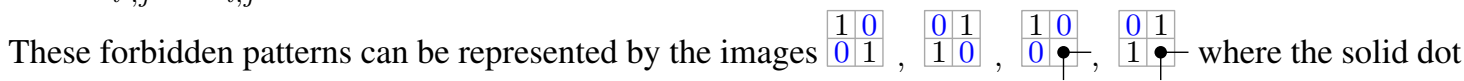
indicates an entry of the permutation.

We say the diagram $E_{w}$ has the south-east (SE) property if whenever $(i, j),\left(i^{\prime}, j\right),\left(i, j^{\prime}\right)$ are in $E_{w}$ with $i^{\prime}>i, j^{\prime}>j$ then $\left(i^{\prime}, j^{\prime}\right)$ is also in $E_{w}$. For such diagrams, condition (ii) in the definition of percentage pseudo-avoidance will never be relevant. Fillings satisfying (i) are called percentage avoiding fillings.

Proposition 2.11. Given any permutation $w$, the following are equal: the number of percentage pseudoavoiding fillings of $E_{w}$, and the number of acyclic orientations of the inversion graph of $w$.

Proof sketch: Consider any 0-1 filling of $E_{w}$. Recall that the elements $\left(i, w_{j}\right)$ of $E_{w}$ are in correspondence with the inversions $(i, j)$ of $w$ and in turn the edges $\{i, j\}$ of $G_{w}$. In the inversion graph $G_{w}$, direct edges corresponding to entries filled with 1 to the right and edges corresponding to entries filled with 0 to the left. One has immediately that the filling of $E_{w}$ contains a percentage pattern if and only if the corresponding orientation of $G_{w}$ contains an alternating 4-cycle (i.e., one whose edges alternate between left and right) and the filling contains a pseudo-percentage pattern (extended using an entry of $w$ ) if and only if the orientation contains a 3-cycle; see Figure 3. Thus, it suffices to show that an orientation of an inversion graph is acyclic if and only if it contains no 3-cycles and no alternating 4-cycles. One implication is obvious. For the other direction, we argue by considering the shortest cycle $C$. If $C$ is a 3-cycle, we are done. If $C$ has length at least 4, one shows first that $C$ is chordless (i.e., there are no edges of $G_{w}$ joinging two vertices in $C$ aside from the edges of $C$ ), second that $C$ is alternating, and finally that $C$ must be an alternating 4-cycle.

Corollary 2.12. If $w$ avoids 321 then the number $\Gamma_{w}$ of $\Gamma$-diagrams on $E_{w}$ is equal to the number of acyclic orientations of the inversion graph of $w$. 

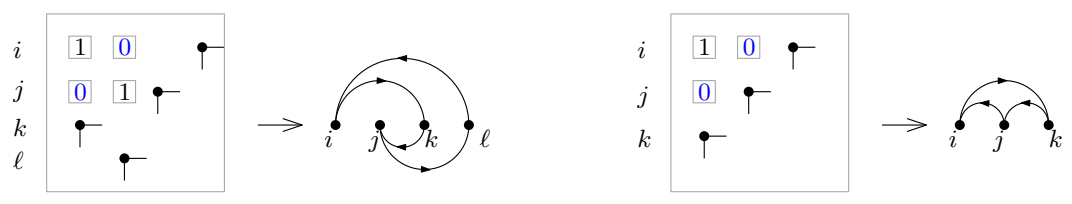

Fig. 3: Left: a percentage pattern in the filling coresponds to a directed 4-cycle in the inversion graph. Right: a pseudo-percentage pattern corresponds to a directed 3 -cycle in the inversion graph.

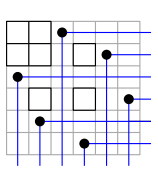

(a)

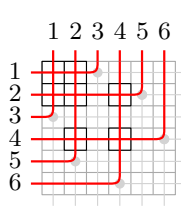

(b)

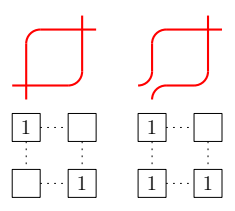

(c)

Fig. 4: (a) The SE diagram of $w=351642$ has $98 \Gamma$-fillings but 100 L-fillings. Postnikov and Spiridonov showed that the latter L-fillings are in bijection with the permutations in the interval [id,351624]. (b) A wiring diagram in $E_{351624}$, (c) the forbidden moves for lex-minimal subwords (in red) and the corresponding L-patterns.

Proof: If $w$ avoids 321 then $E_{w}$ is a skew Young shape in French notation, with some blank rows and columns inserted [7, Prop. 2.2.13]. Thus $E_{w}$ has the SE property and it is easy to see that for such a shape a 0 -1-filling is percentage pseudo-avoiding if and only if it is percentage-avoiding. By work of Spiridonov [11] (see also Josuat-Vergès [4, §4]), the number of percentage-avoiding fillings of such a shape is equal to the number of $\Gamma$-fillings.

Remark 2.13. By Theorem 1.5, if $w$ avoids 321 then the number $\mathrm{L}_{w}$ of L-fillings of $E_{w}$ is equal to the size of the interval $[i d, w]$. (This theorem is due to a nontrivial correspondence between lexicographically minimal subwords of $w$ and L-fillings - see Figure 4) Although the number of L-fillings and $\Gamma$-fillings coincide when $w$ is Grassmannian, these numbers can differ for other 321-avoiding permutations: the permutation 351624 avoids 321, and one can check that there are $98 \Gamma$-fillings on $E_{351624}$ (see Figure 4(a)) and 100 L-fillings on $E_{351624}$.

\section{$3 q$-analogues of rook placements and Bruhat intervals}

In this section we study the relation between a $q$-analogue of $\#[i d, w]$ and a $q$-analogue of $R P_{w}$. The $q$-analogue of \#[id,w] is the Poincaré polynomial $P_{w}(q)=\sum_{u \preceq w} q^{\ell(u)}$ where the order relation in the sum is the strong Bruhat order. The $q$-analogue of the number $R \bar{P}_{w}$ of rook placements we work with is the following: let $\mathbf{F}_{q}$ be the finite field with $q$ elements, and let mat ${ }_{w}(q)$ be the number of $n \times n$ invertible matrices in $\mathbf{F}_{q}$ whose nonzero entries are in $\overline{O_{w}}$. Indeed, $\operatorname{mat}_{w}(q)$ is a $q$-analogue of $R P_{w}$ (see (1) and [6. Prop. 5.1]). The study of invertible matrices over $\mathbf{F}_{q}$ with support avoiding a permutation diagram was initiated in [5] (where the authors used the name "Rothe diagram" for what we call the SW diagram $\left.O_{w}\right)$. 


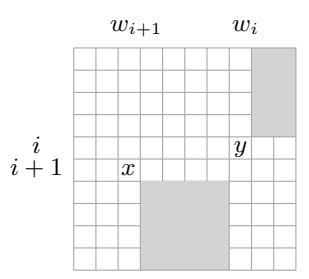

(a)

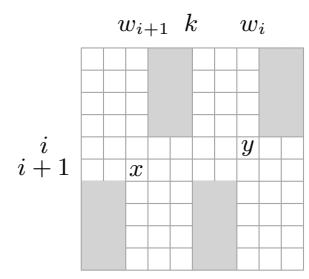

(b)

Fig. 5: (a) A light reduction pair, (b) a heavy reduction pair. The gray areas have no entries $\left(j, w_{j}\right)$ of $w$.

Our proof uses a recursive analysis based on work of Hultman-Linusson-Shareshian-Sjöstrand [3] to prove the following statement (which is one portion of the conjecture [5, Conj. 6.6]).

Theorem 3.1. Let $w$ be a permutation in $\mathfrak{S}_{n}$ and let $O_{w}$ be the $S W$ diagram of $w$. Then $\operatorname{mat}_{w}(q) /(q-$ $1)^{n}=q^{\left(\begin{array}{c}n \\ 2\end{array}\right)+\ell(w)} P_{w}\left(q^{-1}\right)$ if and only if $w$ avoids the patterns $4231,35142,42513$, and 351624 .

The proof of the "only if" part of Theorem 3.1 is as follows: by (1), $\operatorname{mat}_{w}(q) /(q-1)^{n} \equiv R P_{w} \bmod q-$ 1, while we have that $\left.q^{\left(\begin{array}{c}n \\ 2\end{array}\right)+\ell(w)} P_{w}\left(q^{-1}\right)\right|_{q=1}=\#[i d, w]$ by definition of $P_{w}(q)$. If $w$ contains one of the patterns $4231,35142,42513$, or 351624 then by Corollary 2.2, $R P_{w} \neq \#[i d, w]$. Therefore for such $w$ the expressions $\operatorname{mat}_{w}(q) /(q-1)^{n}$ and $q^{\left(\begin{array}{l}n \\ 2\end{array}\right)+\ell(w)} P_{w}\left(q^{-1}\right)$ cannot be equal for sufficiently large $q$.

The "if" part of the proof of Theorem 3.1 is shown by induction, and the rest of this section is devoted to its proof. Let $\mathfrak{S}_{n}(4231,35142,42513,351624)$ be the set of permutations $w$ in $\mathfrak{S}_{n}$ avoiding the patterns 4231, 35142, 42513, and 351624, i.e., the Gasharov-Reiner permutations. We recall the definition of (heavy, light) reduction pairs from [3]. The descent $i$ of $w$ formed by $y=\left(i, w_{i}\right)$ and $x=\left(i+1, w_{i+1}\right)$ is a light reduction pair if there is no entry $\left(j, w_{j}\right)$ with $j<i$ and $w_{j}>w_{i}$, and there is no entry $\left(j, w_{j}\right)$ with $j>i+1$ and $w_{i+1}<w_{j}<w_{i}$. This is illustrated in Figure 5(a) The descent $i$ of $w$ formed by $y=\left(i, w_{i}\right)$ and $x=\left(i+1, w_{i+1}\right)$ is a heavy reduction pair if there is no entry $\left(j, w_{j}\right)$ with $j>i+1$ and $w_{j}<w_{i+1}$, there is no entry $\left(j, w_{j}\right)$ with $j<i$ and $w_{j}>w_{i}$, and there is an index $k$ such that $w_{i} \leq k \leq w_{i+1}$ and there is no entry $\left(j, w_{j}\right)$ with $j<i$ and $w_{i+1}<w_{j} \leq k$ or with $j>i+1$ and $k<w_{j}<w_{i}$. This is illustrated in Figure 5(b).

In [3, Prop. 5.6] it was shown that one can always find a reduction pair in a permutation $w$ in $\mathfrak{S}_{n}(4231,35142,42513,351624)$.

Proposition 3.2 ([3, Prop. 5.6]). Let $w$ be in $\mathfrak{S}_{n}(4231,35142,42513,351624)$. Then either the entries $y, x$ of the first descent of $w$ and/or the entries $\bar{y}, \bar{x}$ of the first descent of $w^{-1}$ form a reduction pair.

In [3] the authors gave recursions for the size of the Bruhat interval below Gasharov-Reiner permutations using the structure imposed by the reduction pairs. In the following sections, we extend this work by giving recursions for the Poincaré polynomial of Gasharov-Reiner permutations, and corresponding recursions for matrix counts. Thus, we will establish by induction that the Poincaré polynomials and matrix counts are essentially equal in this case. 


\subsection{Light reduction pairs}

In this section, we consider the case that the first descent of $w$ is a light reduction pair. The main result of this section is the following:

Proposition 3.3. Let $w$ be in $\mathfrak{S}_{n}$. If the first descent of $w$, involving the entries $y=\left(i, w_{i}\right)$ and $x=$ $\left(i+1, w_{i+1}\right)$, is a light reduction pair then

$$
P_{w}(t)=P_{s_{i} w}(t)+t^{\ell(w)-\ell(w-y)} P_{w-y}(t)
$$

and

$$
\operatorname{mat}_{w}(q)=q \cdot \operatorname{mat}_{s_{i} w}(q)+(q-1) q^{n-1} \cdot \operatorname{mat}_{w-y}(q) .
$$

Proof sketch: In the case of Poincaré polynomials, this result follows from [3, Equation (1)] and [10, Thm. 4]. Thus, we are left to consider the recursion for matrices. We begin by noting a few points about a light reduction pair (see Figure 6(a).

Remark 3.4. Suppose the first descent of $w \in \mathfrak{S}_{n}$ is in position $i$ and is a light reduction pair. Then:

(i) the $i$ th and $(i+1)$ th rows of $O_{w}$ are the same, i.e., they have entries in the same columns; and,

(ii) all the entries in the north-east rectangle $[1, i-1] \times\left[w_{i+1}+1, n\right]$ are in $O_{w}$.

First, we give a convenient interpretation of the term $q \cdot \operatorname{mat}_{s_{i} w}(q)$. Note that $\operatorname{mat}_{s_{i} w}(q)$ counts invertible matrices with support avoiding $O_{w}$ with the additional restriction that the entry at position $z=\left(i+1, w_{i}\right)$ is zero. But from Remark 3.4 (i), this is equivalent to the restriction that the entry at position $y=\left(i, w_{i}\right)$ is zero. Thus, $q \cdot \operatorname{mat}_{s_{i} w}(q)$ counts matrices (possibly singular) with support avoiding $O_{w}$ that become invertible upon setting the $\left(i, w_{i}\right)$-entry equal to 0 .

Second, given a matrix whose support avoids $O_{w} \cup\{y\}$, the submatrix $B$ that results from removing the row and column of $y$ has support in $\overline{O_{w-y}}$ but may or may not be invertible. We use Remark 3.4 (ii) to show that the difference $\operatorname{mat}_{w}(q)-q \cdot \operatorname{mat}_{s_{i} w}(q)$ cancels all the terms where $B$ is not invertible, and that what is left is the product of $(q-1) q^{n-1}$ with the number of choices of invertible $B$. This last term is $\operatorname{mat}_{w-y}(q)$, as desired.

\subsection{Heavy reduction pairs}

In this section, we consider the case that the first descent of $w$ is a heavy reduction pair. We seek to mimic the deletion-contraction method of Section 2. Unfortunately, for a Gasharov-Reiner permutation $w$, the permutation $w^{\prime}$ is not necessarily Gasharov-Reiner: for example, with $w=34512 \in$ $\mathfrak{S}_{5}(4231,35142,42513,351624)$ we have $w^{\prime}=4231$. Thus, given a permutation $w=w_{1} \cdots w_{n}$ whose first descent is a heavy reduction pair in position $i$, let $j$ be minimal such that $w_{i+1}<w_{j}$ and define $v=v(w)$ to be the permutation order-ismorphic to

$$
w_{1} w_{2} \cdots w_{j-1} \quad w_{j+1} w_{j+2} \cdots w_{i} \quad w_{j} \quad w_{i+2} w_{i+3} \cdots w_{n}
$$

in $\mathfrak{S}_{n-1}$. One can show that if $w$ is Gasharov-Reiner then $v$ is a Gasharov-Reiner permutation whose first descent is a light reduction pair. Then the main result of this section is the following:

Proposition 3.5. Let $w$ be in $\mathfrak{S}_{n}$. If the first descent of $w$, involving the entries $y=\left(i, w_{i}\right)$ and $x=$ $\left(i+1, w_{i+1}\right)$, is a heavy reduction pair then

$$
P_{w}(t)=P_{s_{i} w}(t)+t^{\ell(w)-\ell(w-x)} P_{w-x}(t)+t^{\ell(w)-\ell(w-y)} P_{w-y}(t)-t^{\ell(w)-\ell(w-x-y)} P_{w-x-y}(t)
$$




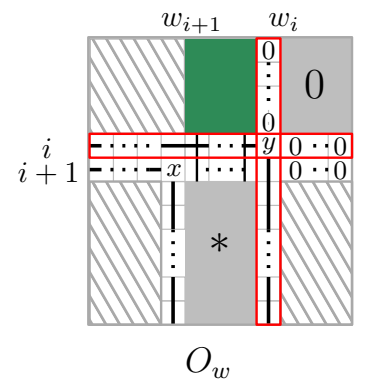

(a)

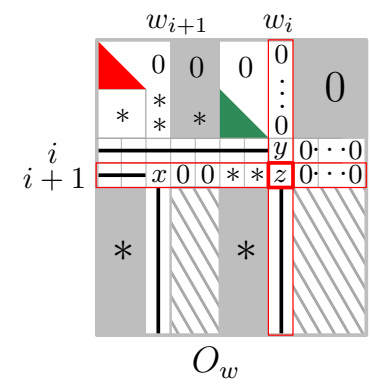

$O_{w}$

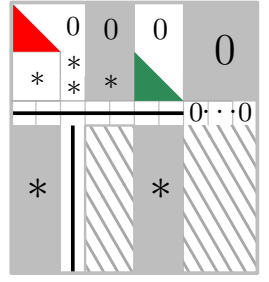

$O_{\operatorname{con}(w)}$

b)

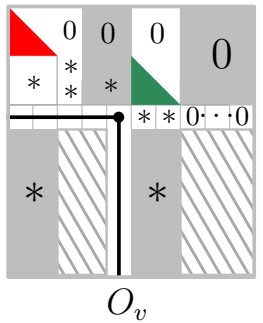

Fig. 6: (a) Decomposition in a light reduction pair. The key features are that the $i$ th and $(i+1)$ th row have entries in the same columns and there is north-east rectangle of zeros. (b) Gaussian elimination with respect to the entry $z$ in a heavy reduction pair. The elimination does not disturb any forced zeros in $O_{w}$.

and

$$
\operatorname{mat}_{w}(q)=\operatorname{mat}_{s_{i} w}(q)+(q-1) q^{n} \cdot \operatorname{mat}_{v}(q)
$$

where $v$ is as in (7).

The main idea of the proof is broadly similar to that of the preceding section: in the case of Poincaré polynomials, we use [10, Thm. 4] to conclude that the argument leading up to [3, Equation (3)] respects the grading of the Bruhat order, and this gives (8). In the case of matrix counts, we use a deletioncontraction argument similar to that of Section 2 by considering whether the entry of a matrix in position $z=\left(i+1, w_{i}\right)$ is zero or nonzero. In the latter case, we show that one can use Gaussian elimination with respect to the nonzero entry in position $z$ without disturbing the zero entries in $O_{w}$.

\subsection{Completing the proof}

Finally, putting the results of this section together we finish the inductive proof of the "if" part of Theorem 3.1

Lemma 3.6. Let $w$ be a permutation in $\mathfrak{S}_{n}$ and let $O_{w}$ be the $S W$ diagram of $w$. If $w$ avoids the patterns 4231, 35142, 42513, and 351624 then $\operatorname{mat}_{w}(q) /(q-1)^{n}=q^{\left(\begin{array}{c}n \\ 2\end{array}\right)+\ell(w)} P_{w}\left(q^{-1}\right)$.

Proof sketch: We induct simultaneously on the size $n$ and the length $\ell(w)$ of the permutation $w$. The result is trivial for identity permutations. Given a nonidentity Gasharov-Reiner permutation $w \in \mathfrak{S}_{n}$, by Proposition 3.2 either the first descent of $w$ or the first descent of $w^{-1}$ is a reduction pair. We have by [5, Prop. 5.2(ii)] that $\operatorname{mat}_{w}(q)=\operatorname{mat}_{w^{-1}}(q)$, and for any $w$ we have $P_{w}(q)=P_{w^{-1}}(q)$, so we can assume that the first descent of $w$ is a reduction pair.

If the first descent of $w$ is a light reduction pair then $\operatorname{mat}_{w}(q) /(q-1)^{n}$ and $q^{\left(\begin{array}{l}n \\ 2\end{array}\right)+\ell(w)} P_{w}\left(q^{-1}\right)$ satisfy the same recursion by Proposition 3.3 . If it is a heavy reduction pair, make a tricky calculation combining Propositions 3.3 and 3.5 to again show that $\operatorname{mat}_{w}(q) /(q-1)^{n}$ and $q^{\left(\begin{array}{c}n \\ 2\end{array}\right)+\ell(w)} P_{w}\left(q^{-1}\right)$ satisfy the same recursion. We conclude by induction. 


\section{Acknowledgements}

We thank Alexander Postnikov for suggesting rooks avoiding permutation diagrams; Ricky Liu, Luis Serrano, Alexey Spiridonov for very helpful discussions; and Pasha Pylyavskyy for the term "zoo" in the introduction. We also thank the developers of FindStat [1], from which we obtained the first evidence for Theorem 2.1

\section{References}

[1] C. Berg, C. Stump, V. Pons, and T. Scrimshaw. Findstat: The combinatorial statistic finder, www . FindStat.org, 2013.

[2] V. Gasharov and V. Reiner. Cohomology of smooth Schubert varieties in partial flag manifolds. J. London Math. Soc. (2), 66(3):550-562, 2002.

[3] A. Hultman, S. Linusson, J. Shareshian, and J. Sjöstrand. From Bruhat intervals to intersection lattices and a conjecture of Postnikov. J. Combin. Theory, Ser. A, 116(3):564-580, 2009.

[4] M. Josuat-Vergès. Bijections between pattern-avoiding fillings of Young diagrams. J. Combin. Theory, Ser. A, 117(8):1218-1230, 2010.

[5] A. Klein, J.B. Lewis, and A.H. Morales. Counting matrices over finite fields with support on skew Young diagrams and complements of Rothe diagrams. J. Alg. Comb., 2013.

[6] J.B. Lewis, R.I. Liu, A.H. Morales, G. Panova, S.V. Sam, and Y.X. Zhang. Matrices with restricted entries and $q$-analogues of permutations. J. Comb., 2(3):355-395, 2011.

[7] L. Manivel. Symmetric functions, Schubert polynomials and degeneracy loci. SMF/AMS Texts and Monographs, 2001.

[8] S. Oh, A. Postnikov, and H. Yoo. Bruhat order, smooth Schubert varieties, and hyperplane arrangements. J. Combin. Theory, Ser. A, 115(7):1156-1166, 2008.

[9] A. Postnikov. Total positivity, Grassmannians, and networks. http://math.mit.edu/ apost/papers/tpgrass.pdf. 2007.

[10] J. Sjöstrand. Bruhat intervals are rooks on skew Ferrers boards. J. Combin. Theory, Ser. A, 114(7):1182-1198, 2007.

[11] A. Spiridonov. Pattern avoidance in Binary Fillings of Grid Shapes. PhD thesis, Massachusetts Institute of Technology, 2009.

[12] J.R. Stembridge. Counting points on varieties over finite fields related to a conjecture of Kontsevich. Ann. Comb., 2(4):365-385, 1998. 